\title{
SÍFILIS NA GESTAÇÃO: ESTRATÉGIAS E DESAFIOS DOS ENFERMEIROS DA ATENÇÃO BÁSICA PARA O TRATAMENTO SIMULTÂNEO DO CASAL
}

\author{
Syphilis in pregnancy: strategies and challenges of nurses of \\ primary care for treat ment couple simultaneous
}

Artigo Original

\section{RESUMO}

Objetivo: Analisar as estratégias e os desafios dos enfermeiros da Atenção Básica para adesão dos parceiros sexuais das gestantes com diagnóstico de sífilis ao tratamento simultâneo da doença. Métodos: Pesquisa qualitativa do tipo exploratório descritiva, desenvolvida com nove enfermeiras da Estratégia Saúde da Família do município de Sobral, Ceará. Os dados foram construídos por meio da entrevista semiestruturada e os resultados foram analisados conforme a técnica da análise categorial temática de Minayo (2010), emergindo duas categorias temáticas: a) Estratégias adotadas pelos enfermeiros para a adesão ao tratamento dos parceiros de gestantes com sífilis; b) Desafios na adesão ao tratamento dos parceiros de gestantes com sífilis. Resultados: Na primeira categoria, as estratégias apontadas pelos enfermeiros para incentivar a adesão ao tratamento dos parceiros sexuais das gestantes com sífilis foram a construção do vínculo, as ações de educação em saúde e a qualificação profissional. Na segunda categoria, os enfermeiros elencaram os desafios na adesão ao dos parceiros das gestantes o desconhecimento sobre a doença, a baixa escolaridade, a precariedade socioeconômica, a exposição de riscos e comportamentos de vulneráveis. Conclusão: Evidenciou-se que os enfermeiros possuem percepção ampla acerca dos fatores que interferem e facilitam para a adesão a dos parceiros ao tratamento de sífilis mas necessitam de melhor embasamento científico e prático para realizarem, de maneira mais eficaz, abordagens aos parceiros sexuais das gestantes com sífilis.

Descritores: Atenção Primária à Saúde; Promoção da Saúde; Assistência de Enfermagem; Cuidado Pré-natal; Sífilis Congênita.

\begin{abstract}
Objective: To analyze the difficulties and strategies of attention of nurses Basic membership to the sexual partners of pregnant women diagnosed with syphilis, the simultaneous treatment of the disease. Methods: A qualitative study of descriptive exploratory, developed with nine nurses of Health of the municipality of Sobral Family Strategy, Ceará. Data were built through semi-structured interviews and the results were analyzed according to the categorical analysis of the technical issue of Minayo (2011), emerging two thematic categories. Results: Point out that the construction of the bond, education actions in health and professional qualification are strategies adopted by nurses for adherence to treatment of partners of pregnant women with syphilis. And as challenges in treatment adherence by partners of professional pregnant elencaram ignorance about the disease, low socioeconomic status, risks and vulnerabilities. Conclusion: It was demonstrated that nurses have extensive perception of aspects of adherence to treatment, but need better scientific and practical basis to perform effectively activities on the management of sexual partners with syphilis.
\end{abstract}

Descriptors: Primary Health Care; Health Promotion; Nursing Care; Prenatal Care; Congenital Syphilis.

\author{
Maristela Inês Osawa \\ Vasconcelos $^{(1)}$ \\ Kilvia Maria Carneiro de \\ Oliveira $^{(1)}$ \\ Ana Hirley Rodrigues \\ Magalhães $^{(1)}$ \\ Raquel Xavier Guimarães ${ }^{(1)}$ \\ Maria do Socorro Carneiro \\ Linhares $^{(1)}$ \\ Maria Veraci de Oliveira \\ Queiroz $^{(1)}$ \\ Izabelle Mont'Alverne N. \\ Albuquerque $^{(1)}$
}

1) Universidade Estadual Vale do Acaraú UVA - Sobral (CE) - Brasil

Este artigo seguiu as normas e formatação
estabelecidas pelo $5^{\circ}$ CIAIQ - Congresso
Ibero-Americano em Investigação
Qualitativa. 


\section{INTRODUÇÃO}

A sífilis congênita (SC) ainda é considerada um importante problema de saúde pública, mesmo sendo uma doença que pode ser evitada por meio da assistência prénatal de qualidade, dos recursos disponíveis e informações adequadas. Entretanto, estudos nacionais e internacionais mostram falhas durante as consultas pré-natais. Do total de casos notificados no Brasil, 75,5\% das gestantes, em 2009, haviam recebido assistência pré-natal, mas apenas 55,4\% tiveram o diagnóstico de sífilis ainda na gestação ${ }^{(1)}$.

Nos últimos 10 anos, houve um progressivo aumento na taxa de incidência de SC: em 2004 a taxa era de 1,7 casos para cada 1.000 nascidos vivos e em 2013 subiu para 4,7. O aumento gradual na notificação de casos na rede de atenção pré-natal, nos últimos anos, deveu-se provavelmente ao fortalecimento dos serviços de pré-natal, por meio da Rede Cegonha, o que propiciou o aumento na cobertura de testagem das gestantes e acompanhamento dos casos. No entanto, apesar da ampliação do diagnóstico, a maioria dos casos continua sendo detectado tardiamente e, consequente aumento das notificações de crianças com $\mathrm{SC}^{(2)}$.

Em 2013, do total de casos do Sistema de Informação de Agravos de Notificações- SINAN, 24,8\% foi notificado no primeiro trimestre de gestação, 31,3\% no segundo trimestre e $36,3 \%$ no terceiro. Quanto às regiões, a Norte apresenta o maior percentual de diagnósticos no terceiro trimestre de gestação, com 50,9\% (29,0\% no segundo e $14,7 \%$ no primeiro) e a Sudeste, o menor, com 31,2\% (30,9\% no segundo e $29,9 \%$ no primeiro). A região Nordeste aparece em segundo lugar, logo após o Norte ${ }^{(2)}$.

No município de Sobral, no Estado do Ceará, conforme dados do SINAN fornecidos pelo serviço de Vigilância Epidemiológica, o município registrou 42 casos de sífilis em gestante durante o primeiro semestre de 2015. No ano de 2014 foram notificados 55 casos e em 201385 casos. Este último foi um dos maiores ocorridos no interior do estado $^{(3)}$.

A SC se dá pela transmissão da bactéria gramnegativa na forma de espiroqueta, denominada de Treponema Pallidum da gestante infectada não tratada, tratada inadequadamente ou reinfectada devido à falta de adesão do parceiro ao tratamento, para o concepto por via transplacentária, podendo ser transmitida em qualquer fase gestacional ou estágio da doença materna.

Observa-se que os principais fatores que induzem ao insucesso da eliminação ou diminuição desse agravo são: as barreiras para o acesso pleno aos serviços de saúde, a falta de solicitação do exame sorológico para gestante durante o pré-natal e a não abordagem para o tratamento e avaliação dos parceiros sexuais das mulheres com resultado positivo.
A Estratégia Saúde da Família (ESF) atua na promoção de ações voltadas aos enfrentamentos dos problemas no processo saúde-doença da população, buscando a longitudinalidade do cuidado aos indivíduos e a prevenção de agravos ${ }^{(4)}$. Nessa estratégia se

insere a atenção pré-natal, a qual tem em seus protocolos triagem da sífilis na gestante e o consequente tratamento da mulher e de seu parceiro, quando apresentam testes positivos. Assim, torna-se um espaço ideal para o controle da sífilis congênita, principalmente no que se refere ao diagnóstico precoce e tratamento adequado dos casos em gestantes com VDRL positivo, bem como de seus parceiros sexuais, que devem receber cuidados concomitantes ${ }^{(5)}$.

Com o tratamento simultâneo do casal, em tempo oportuno do pré-natal, é possível aumentar as chances de minimizar a sífilis congênita. Infelizmente, percebe-se que, mesmo se tendo alta cobertura de pré-natal às gestantes, com média de seis a sete consultas durante a gestação, com a oferta de diagnósticos realizados por meio de técnica simples, rápida e de baixo custo e a disponibilidade da penicilina nas farmácias da ESF, ainda não foram medidas suficientes para promover a eliminação da sífilis congênita como um problema de saúde pública ${ }^{(6)}$.

A enfermagem desempenha um importante papel nas equipes da ESF, pois além de ser responsável por um conjunto ações assistenciais, realiza as consultas de prénatal das gestantes pertencentes às áreas que atua.

$\mathrm{Na}$ assistência ao pré-natal, o enfermeiro deve mostrar a importância do acompanhamento da gestação na promoção da saúde, prevenção e tratamento de distúrbios, durante e após a gravidez e, informá-la dos serviços disponíveis ${ }^{(7)}$.

Étambém durante a consulta de pré-natal, a oportunidade para o enfermeiro promover a educação em saúde com relação aos temas ligados ao ciclo reprodutivo, como o planejamento familiar, sexualidade, DST, amamentação, nutrição e higiene, parto e puerpério. Além das ações de promoção à saúde, deve identificar precocemente riscos para a saúde da gestante e do concepto ${ }^{(8)}$.

Dessa forma, levantou-se a seguinte questão norteadora: Quais as estratégias e desafios enfrentados pelos enfermeiros para que o companheiro/parceiro realize o seu tratamento de sífilis concomitante com a gestante?

Diante à essa realidade e, partindo dos pressupostos de que a SC está relacionada com a qualidade da assistência pré-natal e da existência da interação ativa entre a gestante e o profissional enfermeiro, de modo que ambos troquem saberes e informações visando à promoção do autocuidado, objetivou-se analisar as estratégias e os desafios enfrentados pelos enfermeiros da Atenção Básica para adesão dos parceiros das gestantes com diagnóstico de sífilis, ao tratamento simultâneo da doença. 


\section{MÉTODOS}

Trata-se de uma pesquisa exploratória descritiva com abordagem qualitativa. A perspectiva qualitativa busca entender as percepções que os indivíduos têm da problemática, considerando o lado subjetivo e suas particularidades $^{(9)}$. Já a pesquisa exploratória objetiva gerar maior familiaridade com o problema, com vistas a tornálo mais explícito ou a construir hipóteses ${ }^{(10)}$. A pesquisa foi realizada no município de Sobral, situado no norte do Estado do Ceará, a uma distância de aproximadamente, 220 km da capital, Fortaleza. Possui população estimada para 2015 de 201.756 habitantes, com 63 equipes da Estratégia Saúde da Família, alcançando uma cobertura de mais de 90\% com este tipo de atenção.

O campo de estudo compreendeu seis Centros de Saúde da Família (CSF) que apresentaram mais de três casos registrados de gestantes com sífilis, no primeiro semestre de 2015, segundo dados do SINAN fornecidos pela Vigilância em Saúde ${ }^{(3)}$. Foram incluídos para responderem as questões nove enfermeiras que realizaram atendimento e notificação de casos de sífilis na gestação nesse mesmo período e que aceitaram participar mediante assinatura do Termo de Consentimento Livre e Esclarecido (TCLE). Assim, foram excluídos os enfermeiros dos referidos Centros de Saúde o que não estavam atuando nesse período das notificações. As informações foram coletadas no período de novembro a dezembro de 2015 a partir de entrevista semiestruturada, cujo instrumento foi constituído de perguntas referentes à identificação do perfil das enfermeiras e as perguntas abertas que questionam a abordagem destas profissionais na realização da busca ativa de parceiros sexuais de gestantes com sífilis, bem como, suas impressões sobre aspectos relacionados à adesão dos mesmos aos tratamentos instituídos.

As entrevistas foram gravadas e posteriormente transcritas na íntegra. $O$ tratamento qualitativo das informações foi conduzido a partir da técnica da categorização temática. Foram seguidos três passos: pré-análise, com várias leituras e organização do que foi analisado; exploração do material, com recortes das respostas dos pesquisados e estas agrupadas em categorias; e tratamento dos dados, onde as informações obtidas foram interpretadas $^{(11)}$. Por meio dos dados empíricos foram construídas duas categorias: Estratégias adotadas pelos enfermeiros para a adesão ao tratamento dos parceiros de gestantes com sífilis e Desafios na adesão ao tratamento dos parceiros de gestantes com sífilis.

Esta pesquisa é parte de uma pesquisa maior, que tem como objetivo analisar as dificuldades e estratégias dos enfermeiros da Atenção Básica para adesão dos parceiros das gestantes com diagnóstico de sífilis, ao tratamento simultâneo da doença, no município de Sobral, Ceará, cujo projeto foi analisado e aprovado pelo Comitê de Ética em Pesquisa da Universidade Estadual Vale do Acaraú, sob o CAAE $n^{\circ} 507985159.0000 .5053$ e parecer $n^{\circ} 1.346 .521$

O estudo obedeceu a Resolução do Conselho Nacional de Saúde (CNS) 466/2012 sobre Ética em Pesquisa envolvendo seres humanos. Vale ressaltar que os participantes do estudo foram esclarecidos a respeito do objetivo do estudo, do Termo de Consentimento Livre e Esclarecido, por meio do qual foi obtida a autorização.

\section{RESULTADOS E DISCUSSÃO}

Conforme a caracterização do perfil das nove enfermeiras entrevistadas no estudo, observou-se que a faixa de idade variou entre 24 e 40 anos. Em relação ao tempo de formação, quatro tinham mais de dez anos da conclusão da graduação, sete possuíam especialização, principalmente, em Saúde Pública e Saúde da Família, e uma está cursando. Com relação à participação em algum treinamento sobre sífilis congênita durante a atuação na ESF, do total das enfermeiras entrevistadas, sete relataram ter participado de treinamento ou atividade de Educação Continuada sobre SC realizada pela Secretaria Municipal de Saúde de Sobral.

Após a pré-análise das informações obtidas, ou seja, leituras e organização das falas das enfermeiras pôde-se realizar um recorte dessas falas e estabelecer as seguintes categorias para a discussão dos resultados: Estratégias adotadas pelas enfermeiras para a adesão ao tratamento dos parceiros de gestantes com sífilis; b) Desafios na adesão ao tratamento dos parceiros de gestantes com sífilis.

Diante dos depoimentos das enfermeiras, foi possível apreender a importância dada ao aconselhamento, na Estratégia Saúde da Família do município, bem como adequada conduta seguida por estas profissionais, após detectar o caso de sífilis, conforme as recomendações ministeriais.

Constata-se que as respostas fornecidas estão de acordo com os protocolos, ou seja, notificar o caso, dar início imediato ao tratamento da DST para o casal, orientando-os sobre a importância do tratamento para eles e para o seu filho, procurando abordar o assunto de forma clara.

Ressalta-se que cada profissional tem a sua forma peculiar em conduzir a situação, exemplo disso é o uso de tecnologias leves como o aconselhamento, quanto à adesão ao tratamento, o uso de preservativo durante a relação e incentivo em continuar acompanhando o pós-tratamento, através dos exames do VDRL quantitativo, em que se avalia a titulação. Relatam que inicialmente, realizam o aconselhamento do parceiro de forma individual e sigilosa, 
na ausência de sua companheira nesse primeiro contato com a profissional de saúde, evitando constrangimentos e fazendo sentir-se mais acolhido naquele ambiente que vai precisar frequentar.

"Realizar abordagem e tratamento mais acompanhamento pós-tratamento. No caso a abordagem individual, trabalhando politica de Redução de Danos juntamente com a corresponsabilidade e autonomia do sujeito". (Enf. 6)

"Conversar com o casal. Falar sobre a importância do tratamento de ambos e avisar sobre possiveis consequências para o bebê e para os pacientes futuramente." (Enf. 2).

"Solicitar a presença do Parceiro à Unidade para aconselhamento quanto à adesão ao tratamento e o uso de preservativo.", (Enf. 7)

O enfermeiro, ao aconselhar o parceiro da gestante, deve proporcionar um acolhimento adequado, transmitindo confiança e pressupondo um ambiente profissional e agradável, no qual o cliente tenha garantia de sigilo, privacidade, confidencialidade no atendimento ${ }^{(12)}$.

As ideias expostas pelas enfermeiras ajudam a compreender de forma satisfatória os depoimentos a respeito das estratégias que adotam para adesão dos homens ao tratamento da sífilis. Elas buscam conscientizálos da importância do tratamento da doença enquanto estão sensíveis pela expectativa de tornarem pais e tomam como ponto de partida de que precisam "se cuidar para poder cuidar da família".

O aconselhamento é uma das estratégias propostas pelo Ministério da Saúde para o controle e quebra da cadeia da transmissão da sífilis adquirida, que além das consequências provocadas à saúde do indivíduo adulto, uma gestante infectada, e não tratada adequadamente, pode transmitir a doença para o seu concepto, por via transplacentária.

Na Atenção Básica, o aconselhamento é uma atividade realizada pelos profissionais de saúde e busca efetivar ações de prevenção em DST, oportunizando a um diálogo, no que diz respeito às práticas íntimas relacionadas com o simbolismo social e cultural determinadas pela sociedade carregadas de preconceitos, e que não auxiliam no entendimento da doença.

Nos momentos do aconselhamento é possível identificar os recursos pessoais e sociais que auxiliam na adesão ao tratamento, bem como na organização de um plano factível de redução de riscos, baseado na realidade e nas possibilidades do usuário. Isto ocorre devido a avaliação do risco ser trabalhada no atendimento individual, em que o profissional de saúde precisa estar atento as suas próprias opiniões, ao possibilitar ao usuário se expressar abertamente sem juízo de valor ${ }^{(13)}$.
Na opinião das enfermeiras a construção de vínculo que estabelecem com os usuários é de fundamental importância para a melhoria do atendimento pois, é a partir desse componente que se estabelece uma relação de confiança com o usuário no cotidiano. $\mathrm{O}$ vínculo, na opinião das entrevistadas, amplia a eficácia do tratamento, favorecendo a participação do parceiro nas ações desenvolvidas por meio de uma boa acolhida e uma escuta qualificada.

"Após estabelecermos o vínculo com o portador da patologia, a abordagem, bem como a continuidade do tratamento torna-se flexivel e você obtém bons resultados na adesão" (Enf. 7).

"Sim, pois de uma forma geral conseguimos realizar uma abordagem, já que possuímos um bom vínculo" (Enf.1).

"Estabelecer empatia, boa comunicação ao esclarecer beneficios do tratamento tanto para o companheiro, como para a gestante e seu futuro filho" (Enf. 4).

A comunicação eficaz, mencionada pelas enfermeiras, tem como prioridade evitar falhas e valorizar o encontro com parceiros de gestantes com sífilis, além de buscar iniciativas que favorecem o vínculo. Acreditam que os acordos e responsabilidades compartilhadas podem ser traçadas com êxito no cuidado em saúde.

Os depoimentos confirmam o mérito do vínculo entre comunidade e as enfermeiras ao realizar a assistência pré-natal. De acordo com a Política Nacional de Atenção Básica (PNAB), o vínculo tem a finalidade de estabelecer relação de afetividade entre profissional e usuário, família, comunidade, baseada na ética, na corresponsabilidade, no respeito e na confiança. A construção do vínculo não depende apenas da equipe de saúde, mas também dos usuários por carregarem em si um potencial terapêutico. A base do vínculo para a equipe é o compromisso com a saúde daqueles que a procuram, ou são por ela procurados ${ }^{(14)}$.

Portanto, o acolhimento e vínculo são decisivos na relação de cuidado entre trabalhador da saúde e o sujeito, permeando todas as fases do tratamento. Facilita a construção da autonomia mediante a responsabilização compartilhada e pactuada entre os sujeitos envolvidos na terapêutica, propiciando um cuidado integral ${ }^{(15)}$.

A partir dos depoimentos percebeu-se que, as formas de planejar e executar as ações implicam que o casal esteja unido na adesão ao tratamento das DST. As ações de educação em saúde com a utilização de materiais educativos, como álbuns seriados abordando informações sobre DST, têm impacto positivo na compreensão sobre a doença, favorecendo o conhecimento, o que pode induzir mudanças de comportamentos. 
“Oriento riscos, tratamento e riscos ao feto" (Enf. 3).

"Oriento as consequências para o feto, em caso de não adesão ao tratamento " (Enf. 1).

"Conscientização, sensibilização, orientações quanto a importância do tratamento para o bem estar da gestação", (Enf. 9).

“Apresentando álbum seriado de DST, então gera impacto no paciente e possíveis consequências futuras, caso não venha a fazer tratamento" (Enf. 4).

"Primeiro precisa-se esclarecer o caso em questão, abordando os riscos de sifilis na gestação. Depois, abordando o que é a doença, sintomas clínicos e riscos. A UBS possui álbum seriado para este fim” (Enf. 5).

[...] "fazemos aquela abordagem bem impactante" (Enf. 4).

Nas abordagens com os parceiros sexuais de gestantes com sífilis, as enfermeiras desenvolvem ações proativas de educação em saúde, o que contribuem significativamente para a sua adesão ao tratamento da sífilis adquirida. As orientações são realizadas com a utilização de materiais educativos que enfocam a importância do tratamento simultâneo do casal, com vistas à prevenção da transmissão vertical na gestação atual e futura.

$\mathrm{O}$ enfermeiro como orientador precisa identificar os fatos e instigar os usuários quanto às lacunas do conhecimento sobre determinados assuntos, principalmente se estes estão relacionados com o seu estado de saúde. Nos casos dos indivíduos com sífilis, a desempenho como educador é importante, uma vez que é o profissional de saúde mais apto a fornecer orientações a gestante e seu parceiro, porque além de possuir os conhecimentos científicos necessários, pode estabelecer, na maioria das vezes, uma relação de confiança com eles, esclarecendo-os sobre a evolução clínica da sífilis, forma de transmissão e de prevenção ${ }^{(16)}$.

As instruções normativas do Ministério da Saúde para o controle e prevenção da sífilis direcionam ações para que profissionais, ao atenderem a gestante com sífilis e seu parceiro, assumam uma postura acolhedora e não julgadora; identifiquem as crenças e os valores dos clientes acerca das DST, e utilizem linguagem compatível com a cultura dos $\operatorname{mesmos}^{(12)}$.

Nesse contexto, educação em Saúde é um processo de pensar e fazer emancipatório de interesse da pessoa e coletividade, portanto, instrumento essencial do trabalho de cuidar em Enfermagem, que deve envolver profissionais comprometidos para assegurar prática educativa em saúde que envolva a família e a comunidade ${ }^{(17)}$.

Desse modo, é necessário reinventar a prática de enfermagem utilizando recursos criativos e imaginativos. $\mathrm{O}$ diálogo e a utilização de imagens e textos sobre determinada patologia, despertam nos usuários um olhar diferente sobre a doença, que dependendo do estágio é assintomática, mas requer atenção e cuidado ${ }^{(18)}$.

As enfermeiras do estudo, também, deram ênfase à qualificação profissional como estratégia na garantia de adesão ao tratamento. Suas falas evidenciaram que se sentem treinadas e preparadas na realização da abordagem do parceiro sobre a condição de portador de sífilis. Segundo as mesmas, alguns fatores contribuíram para esta qualificação, por exemplo, os treinamentos em serviços que participaram no Centro de Orientação e Apoio Sorológico de Sobral (COAS) e os oferecidos como Educação Permanente pela Escola de Formação em Saúde da Família Visconde de Sabóia de Sobral. Estes treinamentos foram importantes e lhes ajudaram a melhor conduzir situações de aconselhamento para DST.

"Sim, o município em questão realizou capacitações nos preparando para atuar nos casos de gestantes e parceiros com sifilis (Educação Permanente) descentralizada, no caso COAS para este fim”. (Enf. 5)

"Sim, devido as informações em treinamentos (Enf. 2)

"Sim, devido o protocolo de sifilis, pois conduzimos o tratamento e a abordagem da gestante e do parceiro. “ (Enf. 6)

Considerando a complexidade da abordagem aos parceiros de gestantes com sífilis, as participantes consideraram que a qualificação profissional é um fator facilitador para a melhoria na abordagem, sensibilização e tratamento dos parceiros. Os momentos de educação permanente e capacitações desenvolvidas pela Secretaria Municipal de Saúde de Sobral contribuem para a adequada condução do tratamento do homem portador de sífilis.

A educação permanente, parte do pressuposto da aprendizagem significativa (promove e produz sentidos) e propõe que a transformação das práticas profissionais deva basear-se na reflexão crítica sobre as ações concretas dos profissionais na rede de serviços. Propõe que os processos de capacitação do pessoal da saúde sejam estruturados, a partir da problematização do seu processo de trabalho e que tenham como objetivo a transformação das práticas profissionais e da própria organização do trabalho ${ }^{(19)}$.

\section{Desafios na adesão ao tratamento dos parceiros de gestantes com sífilis}

Nopresenteestudo, asenfermeirascolocaramemdestaque alguns desafios para a adesão ao tratamento relacionado aos parceiros de gestantes, como: desconhecimento da doença, baixa condição socioeconômica, riscos e vulnerabilidades, terapia medicamentosa e seguimento do tratamento. 
Em relação ao tratamento, a terapia medicamentosa por via intramuscular contribui para a resistência à dor na administração e à quantidade de doses necessárias para a conclusão do tratamento. Esta condição foi relatada por uma enfermeira, enquanto outra considerou que o tratamento é bem aceito, porém, o que dificulta é a realização do exame VDRL quantitativo mensal, importante para o seguimento.

"A dor da injeção Penicilina Benzatina, em três semanas seguidas dificulta o tratamento (Enf. 2)

"O tratamento em si é de boa aceitação. O desafio maior é realizar o acompanhamento mensal VDRL quantitativo para gestante. " (Enf. 5)

O desconforto gerado pela aplicação intramuscular da penicilina benzatina influencia na aderência ao tratamento. Deve ser tentado alternativas de tratamento com outras drogas que, de preferência, deveria ser ministrado em dose única, visto que o seu objetivo é a quebra imediata da cadeia de transmissão ${ }^{(20)}$.

Ressalta-se a importância fazer explicação explicar aos parceiros das gestantes com sífilis sobre a via de administração, o local, a quantidade de doses a ser tomada e os profissionais tranquilizarem sobre a dor e esclarecer dúvidas sobre o medo de uma possível reação medicamentosa adversa, além de registrar na caderneta da gestante os dias em que as doses foram administradas.

Compreende-se que a doença, durante a gestação, só é adequadamente tratada, sem danos ao feto, quando se realiza a administração da penicilina benzatina com doses apropriadas para o estágio da infecção. O tratamento é concluído em pelo menos trinta dias antes do parto, e o parceiro tratado simultaneamente com o mesmo esquema terapêutico da gestante ${ }^{(21)}$.

Os depoimentos das enfermeiras revelam que o conhecimento inadequado das gestantes e seus parceiros também podem estar associados ao seu nível de escolaridade. Outra questão é que, o pouco conhecimento das mulheres quanto à sífilis, pode ter sido adquirido somente após a confirmação da doença. Isso leva-se a acreditar que antes do diagnóstico as mulheres não possuíam conhecimento algum acerca do problema.

"Paciente leigo, com pouca orientação sobre a doença, de difícil adesão, sempre dificulta para o tratamento." ( Enf. 6)

"Gestante e parceiro que não reconhecem no tratamento uma forma de evitar a disseminação para o feto"(Enf. 9)

Por uma série de razões, as gestantes e parceiros continuam sem saber o que é a doença, como ocorre a transmissão da sífilis e a existência de prevenção para ela. Como a sífilis, em muitos casos, se apresenta assintomática, ela não é vista como uma doença, dificultando assim o tratamento e entendimento sobre sua transmissão ${ }^{(22)}$.

A baixa escolaridade dos indivíduos pode ter efeito na percepção dos problemas de saúde e na capacidade de entendimento das informações nessa área, como também no consumo e na utilização dos serviços de saúde e na adesão aos procedimentos terapêuticos ${ }^{(2)}$.

É importante destacar a possibilidade de que as gestantes e parceiros tenham sido informados sobre a doença e este resultado sugere a necessidade de reavaliar as estratégias educativas que, ao longo da história, vêm sendo reproduzidas dentro do setor saúde. Também é de se esperar que populações pobres, com baixo nível de escolaridade, tenham dificuldades de apreender informações ${ }^{(23)}$.

A aceitação do parceiro sexual na adesão ao tratamento para sífilis possui uma configuração multifatorial, envolvendo aspectos relacionados ao nível socioeconômicocultural e educacional, incluindo até as questões vinculadas ao próprio tratamento, tais como medicação e a unidade de saúde, na qual a medicação é administrada ${ }^{(24)}$.

A adesão do parceiro, bem como seu comportamento guarda uma relação direta com o sucesso do tratamento, pois mulheres que tem o apoio do parceiro apresentam cinco vezes mais chances de concluírem o tratamento, devido o apoio que recebem ${ }^{(6)}$. Infelizmente, de acordo com os depoimentos declarados neste estudo, em alguns CSF de Sobral acontece situação contrária, isto é, existem parceiros que não apoiam a gestação, desafiando o trabalho dos profissionais.

\section{"Falta de apoio do parceiro na gestação. " (Enf. 7)}

"Desafio social (mãe solteira e múltiplos parceiros), desconhecimento da DST, preconceito social, gestações não planejadas, sigilo das informações dos parceiros." (Enf. 3)

"Uso de álcool e drogas, profissionais do sexo com companheiros caminhoneiros" (Enf. 4)

Em pesquisas realizadas sobre o assunto no Brasil, é baixo o número de parceiros de gestantes com sífilis que comparece aos serviços de saúde para realizar o tratamento. Isto acontece devido ao fato da grande parte da sociedade carregar preconceito e ainda ter uma visão machista de que a gravidez e a criação dos filhos são de responsabilidade exclusiva das mulheres, o que contribui para o aumento deste quadro negativo no país ${ }^{(25)}$.

$\mathrm{Na}$ opinião das enfermeiras, em Sobral, existem algumas barreiras para o acesso dos parceiros das gestantes que trabalham em horário que coincide com o do funcionamento do CSF. Outros não frequentam o CSF por não terem relacionamentos estáveis com as gestantes e 
não se sentem responsáveis pela relação constituída com a gestante.

Um estudo realizado na cidade do Rio de Janeiro, os parceiros de gestantes com relacionamento estável, apresentam 24 vezes maiores chances de adesão ao tratamento em comparação aos parceiros de relacionamentos casuais $^{(6)}$.

Estas situações se destacam como desafios para as enfermeiras na busca dos companheiros das gestantes com sífilis para realizarem o tratamento.

\section{CONSIDERAÇÕES FINAIS}

Estudo indicou as estratégias das enfermeiras da atenção básica e os desafios para a adesão ao tratamento de parceiros sexuais de gestantes com sífilis. Para as participantes, o aconselhamento, a qualificação profissional contínua e a construção do vínculo estabelecido com o usuário facilita o acesso ao serviço, a adesão no tratamento e o tratamento adequado.

As gestantes e parceiros vivenciam dificuldades associadas ao contexto social no qual estão inseridos, como a precariedade socioeconômica, a baixa escolaridade, a multiplicidade de parceiros, o uso de substâncias ilícitas e o desconhecimento acerca da doença. O pouco conhecimento acerca da sífilis por parte das gestantes e dos parceiros foi um fato unânime nos depoimentos das enfermeiras. $\mathrm{O}$ conhecimento é inerente à prevenção e à adesão ao tratamento da doença e o seu desconhecimento torna a problemática da sífilis ainda maior, ocasionando sentimentos e atitudes que dificultam o processo de cura e prevenção da doença.

Dessa forma, infere-se que, a prevenção da sífilis congênita se cerca pela abordagem aos parceiros sexuais das gestantes com sífilis e da garantia da realização do tratamento adequado do casal. Estes são grandes desafios para os profissionais da atenção básica. Entretanto, ainda, são necessárias ações mais incisivas para a sua prevenção, incluindo, além da notificação de todos os casos, a busca ativa, o diagnóstico e tratamento precoce, realizado de maneira adequada, o seguimento e o acompanhamento sorológico até a comprovação da cura.

A atenção de enfermagem qualificada às gestantes com sífilis, além de garantir sua saúde no período gestacional e do seu concepto, tem por finalidade, oferecer uma atenção integral no intuito de curá-la da infecção, prevenir a sífilis congênita no seu filho, apoiar e acolher o seu companheiro para sua inserção no tratamento da sífilis.

Este estudo oportunizou as autoras algumas questões para reflexão acerca dos desafios que os profissionais de saúde enfrentam para a obtenção de resultados positivos na prevenção da sífilis congênita, entre eles a adesão dos parceiros sexuais das gestantes com sífilis ao tratamento no tempo oportuno.

\section{REFERÊNCIAS}

1. Brasil. Ministério da Saúde, Coordenação de Doenças Sexualmente Transmissíveis e Aids. Sífilis: Estratégias para Diagnóstico no Brasil. Brasília: Ministério da Saúde; 2010.

2. Brasil. Secretaria de Vigilância em Saúde, Departamento de DST, Aids e Hepatites Virais. Boletim Epidemiológico - Sífilis. Brasília: Ministério da Saúde; 2015.

3. Sobral. Sistema de Informação de Agravos de Notificação, Boletim epidemiológico- Sífilis. Sobral: Secretaria Municipal de Saúde; 2015.

4. Brasil. Secretaria de Vigilância em Saúde, Política Nacional de Promoção da Saúde. Brasília: Ministério da Saúde; 2006.

5. Saraceni V, Angélica EM. Relação entre a cobertura da Estratégia Saúde da Família e o diagnóstico de sífilis na gestação e sífilis congênita. Cadernos de Saúde Pública. 2012; 28(3): 490-496.

6. Hildebrand, VLPC. Sífilis congênita: fatores associados ao tratamento das gestantes e seus parceiros [dissertação]. Rio de Janeiro: Escola Nacional de Saúde Pública Sérgio Arouca: Escola Nacional de Saúde Pública Sérgio Arouca; 2010.

7. Barbosa, TL, de A, Gomes, LMX, Dias, OV. O prénatal realizado pelo enfermeiro: a satisfação das gestantes. CogitareEnferm.2011; 16(1): 29-35.

8. Santos EV. A importância do pré-natal e o papel do enfermeiro neste contexto. Portal Educação. [periódico na internet]. 2014 [acesso em 2016 Dez 10]; Disponível em: https://www.portaleducacao.com. br/enfermagem/artigos/57913/a-importancia-do-prenatal-e-o-papel-do-enfermeiro-neste-contexto

9. Bell J. Projeto de pesquisa. Guia para pesquisadores iniciantes em educação, saúde e ciências sociais. Porto Alegre: Artmed; 2008.

10. Gil AC. Como elaborar projetos de pesquisa. São Paulo: Atlas; 2010.

11. Minayo MCS. O desafio do conhecimento: pesquisa qualitativa em saúde. São Paulo: Hucitec; 2010.

12. Guerreiro EM, Rodrigues DP, Silveira MAM, Lucena, NBF. O cuidado pré-natal na atenção básica de saúde 
sob o olhar de gestantes e enfermeiros. Rev REME. [periódico na internet]. 2011 [acesso em 2016 Jan 10]; 16(3). Disponível em: http://reme.org.br/artigo/ detalhes $/ 533$

13. Dantas AKC, Santos FAPS, Mulatinho AS, Carvalho JBL, Silva GE. Adesão do parceiro ao tratamento da sífilis na gestação: uma contribuição do Enfermeiro para o impacto sobre a sífilis congênita [abstract]. In: II Congresso Internacional de Enfermagem Obstétrica e Neonatal; 2013 Out 30- 31, Nov 1º; Florianópolis Santa Catarina; 2013.

14. Brasil. Coordenação de Vigilância, Informação e Pesquisa, Boletim epidemiológico Sífilis. Brasília: Ministério da Saúde; 2012.

15. Jorge MSB, Pinto DM, Quinderé PHD, Pinto AGA, Sousa FSP, Cavalcante CM. Promoção da Saúde Mental - Tecnologias do Cuidado: vínculo, acolhimento, corresponsabilização e autonomia. Ciênc Saúde Coletiva. 2011; 16(7): 3051-3060.

16. Potter PA, Perry AG. Fundamentos de enfermagem. Rio de Janeiro: Elsevier, 2013.

17. Leite CT, Vieira RP, Machado CA, Quirino GS, Machado MFAS. Prática de educação em saúde percebida por escolares. Cogitare Enferm. 2014; 19(1): $13-26$.

18. Almeida Filho AJ, Moraes AEC, Peres MAA. Atuação do enfermeiro nos centros de atenção psicossocial: implicações históricas da enfermagem psiquiátrica. Rev. Rene. 2009; 10(2): 158-65.

19. Brasil. Secretaria de Atenção à Saúde, Departamento de Atenção Básica. HIV/Aids, hepatites e outras DST. Brasília: Ministério da Saúde; 2005.
20. Brasil. Secretaria de Vigilância em Saúde, Programa Nacional de DST/ Aids. Manual de Controle de Doenças Sexualmente Transmissíveis. Brasília: Ministério da Saúde; 2006.

21. Magalhães DMS, Kawaguchi IAL, Dias AC, Paranhos IM. Sífilis materna e congênita: ainda um desafio. Cadernos de Saúde Pública. 2013; 29(6): 1109-20.

22. Cavalcante AES, Silva MAM, Rodrigues ARM, Mourão Netto JJ, Moreira ACA, Goyanna NF. Diagnóstico e Tratamento da Sífilis: uma Investigação com Mulheres Assistidas na Atenção Básica em Sobral, Ceará. DST J Bras Doenças Sex Transm. 2012; 24(4):239-45.

23. Silva MRF, Brito ESV, Freire LCG, Pedrosa MM, Sales VMB, Lages I. Percepção de mulheres com relação à ocorrência de sífilis congênita em seus conceptos. Rev APS. 2010; 13(3): 301-09.

24. Figueiredo MSN, Cavalcante EGR, Oliveira CJ, Monteiro MFV, Quirino GS, Oliveira DR. Percepção de enfermeiros sobre a adesão ao tratamento dos parceiros de gestantes com sífilis. Rev Rene. 2015;16(3):345-54.

25. Santos VC, Anjos KF. Sífilis: uma realidade prevenível. Sua erradicação, um desafio atual. Revista Saúde e Pesquisa. 2009;2(2):257-63.

\section{Endereço para correspondência:}

Maristela Inês Osawa Vasconcelos

Rua Balbino, 328

Bairro: Campo dos Velhos.

CEP: 62040-200 - Sobral - CE - Brasil

E mail: miosawa@gmail.com 